\title{
V009 LINEARIZED TARGET-ORIENTED INVERSION - APPLICATION TO REAL DATA
}

\author{
G. BERNASCONI, G. DRUFUCA and F.L. ROCCA \\ 20133 Milan, Italy
20 Ilazza Leonardo da Vinci 32,
}

We have developed a procedure for target-oriented linearized elastic inversion in the $k$ - $\omega$ domain (Bernasconi et al., 1994), exploiting to the limit the weak scatter hypothesis.

The technique is based upon two separate phases: linearized seismic inversion (limited to the target) and the description and compensation of propagation effects through the overburden (datuming).

Limiting the inversion to a specific depth interval (target zone) improves the efficiency of the technique. Operating in the linear approximation in the $k$ - $\omega$ domain we gain the advantage of the simplicity of the theory and the capability of controlling the negative effects on resolution of acquisition geometry and limited bandwidth: ambiguities are carefully avoided by inverting only well conditioned components. We obtain an image of the target that has tendencially minimum contributions of errors from lack of experimental resolution: this "seismic" image can be used as initial macromodel for interpretation, techniques of lateral continuation of well information, petrophysical and geostatistical analysis, $4 \mathrm{D}$ surveys.

The technique is successfully demonstrated on the Mobil data set (Keys and Foster, 1995). The seismic line consists of 1001 shot records. Each shot record was recorded on 120 channels for six seconds. Two wells (well A and well B) intersect the seismic line. Well log data are available. We have selected a target zone located at a depth of $2 \mathrm{~km}$, centred on CDP 808 (well A) where well data exhibits an increase of oil content. Processing is limited to a corresponding portion of the whole data set, ranging from CDP 660 to CDP 1130. It covers an horizontal range of $5875 \mathrm{~m}$. Water bottom multiples are a major source of noise. There is also evidence of interbed multiples and P-SV conversions (Fig. 2). The main steps to be performed are: (1) macro model estimation; (2) multiple and conversion elimination; (3) datuming to bring the data from the surface to the target level; (4) linearized elastic inversion of the target.

We have used well data to constrain the picking in the velocity analysis and to infer an empirical depth-dependent relation between $\mathrm{P}$ and $\mathrm{S}$ velocities. This relation gives an estimate of the $\mathrm{S}$ velocity macromodel. A similar approach has been followed for the lateral continuation of density. We have performed an $f-k$ filtering to remove multiples and conversions. As an example Fig. 1 shows CDP gather 808 (Well A) before (Fig. 1a) and after the processing (Fig. 1b). Figs. 1c and 1d show the corresponding semblance panels: the effect of multiples is visible. Fig. 2 shows the final estimated macro model for $P$ velocity and the selected target zone.

A qualifying feature of our inversion scheme is to be limitable to the target zone (Bernasconi et al., 1994). This requires the datuming of the sources and receivers on the surface to the target level. It ideally removes the transmission effects of the overburden. 
Our datuming code (Bernasconi et al., 1995) uses plane waves transfer functions of the overburden, computed tracing Gaussian beams through the macromodel: as an example Fig. 2 shows the geometry for the ray tracing process and some shots.

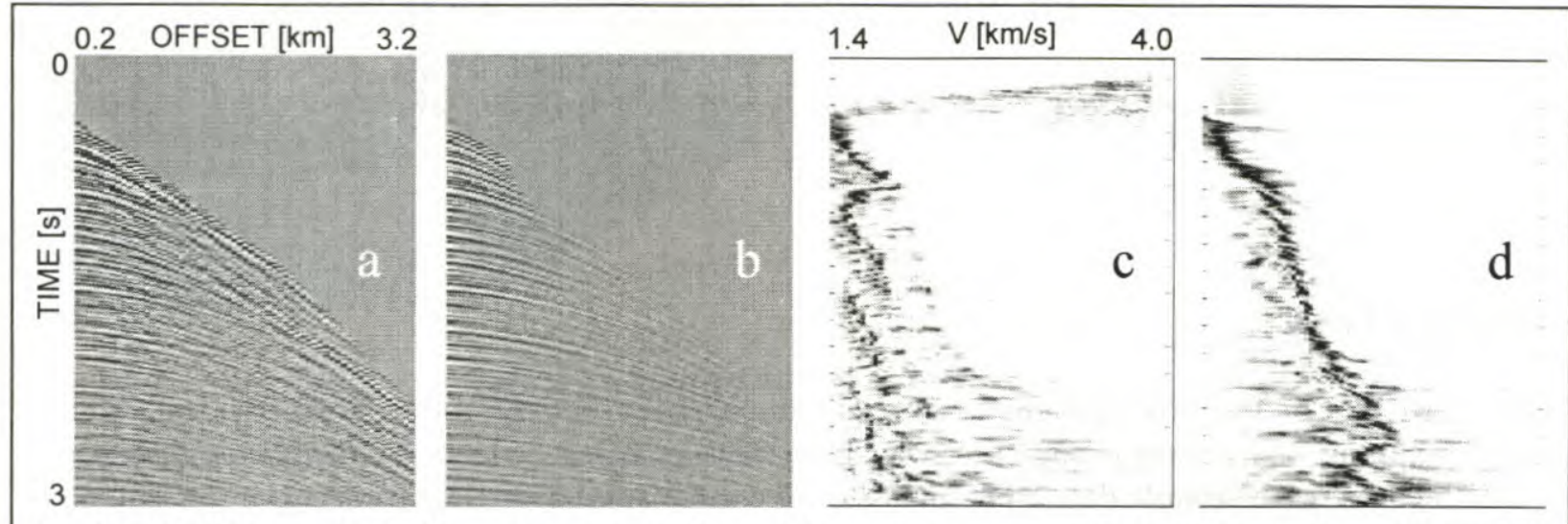

Fig. 1.CDP808 (well A). Raw data (a) and semblance (c); processed data (b) and semblance(d).

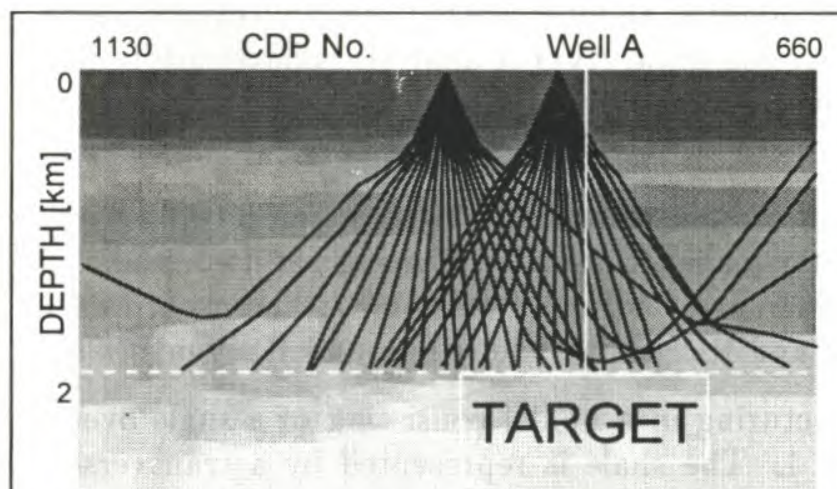

Fig. 2. P velocity macro model and ray tracing for the datuming process with some shots.
Datumed data are finally inverted: fig. 3 shows the P-impedance relative perturbation in the target zone. Superimposed is the P-impedance relative perturbation evaluated from well data after blocking and spatial filtering. The general agreement is excellent and for an unusually long depth interval of $500 \mathrm{~m}$. This validates the global procedure including datuming and inversion.

We acknowledge Mobil Oil for releasing this data set. Thanks also to G. Di Pasquale and A. Mobili for processing the data and to N. Bienati for helping with the software. This research is sponsored by EC within the framework of the GEOSCIENCE II Program, Contract JOU2-CT92-0096.

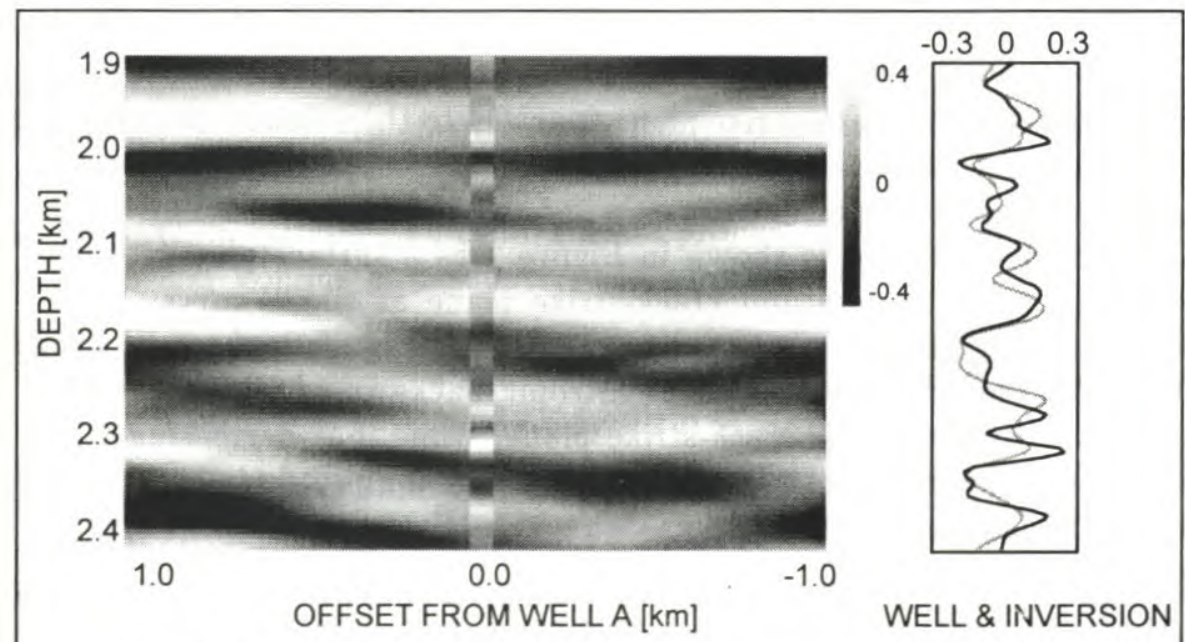

Fig. 3. Inverted P-impedance perturbation and well data.

\section{References.}

Keys, R.G., Foster, D.J., 1995: A data set for evaluating and comparing seismic inversion methods. Proceeding of the 1994 SEG Workshop on the Mobil Data Set, Los Angeles.

Bernasconi,G.,Drufuca, G., Rocca, F., 1994: Linearized elastic inversion in the $k-\omega$ domain. Extended Ab-

stracts of the 56th EAEG Meeting, Vienna, Austria.

Bernasconi, G., Drufuca, G., Rocca, F., 1995: Datuming for target-oriented inversion in the $k$ - $\omega$ domain. Extended Abstracts of the 57th EAEG Meeting, Glasgow, UK. 